\title{
"STA-MCA bypass with encephalo-duro-myo-synangiosis combined with bifrontal encephalo-duro-periosteal-synangiosis" as a one-staged revascularization strategy for pediatric moyamoya vasculopathy
}

\author{
Esposito, Giuseppe ; Kronenburg, Annick ; Fierstra, Jorn ; Braun, Kees P J ; Klijn, Catharina J M ; \\ van der Zwan, Albert ; Regli, Luca
}

\begin{abstract}
PURPOSE Moyamoya vasculopathy progressively compromises cerebral blood flow resulting in chronic hypoperfusion. The middle cerebral artery (MCA) territory and the bifrontal areas are the regions most frequently affected. Although most techniques aim to only revascularize the MCA territory, augmentation of blood flow of the bifrontal areas is of importance in the pediatric moyamoya population since these regions play an important role in cognition, intellectual development, and in lower extremity and sphincter function. We recently described a one-staged surgical procedure combining revascularization of three regions, the MCA territory unilaterally and the frontal areas bilaterally. The purpose of this article is to report our surgical experience in eight children and to emphasize the rational for bifrontal revascularization. METHODS We report a case series consisting of eight children where the following surgical strategy was applied: (1) a direct superficial temporal artery-to-middle cerebral artery (STA-MCA) bypass with encephalo-duro-myo-synangiosis (EDMS) for unilateral MCA revascularization; in combination with (2) a bifrontal encephalo-duro-periosteal-synangiosis (EDPS) for bifrontal revascularization. Patients' characteristics and 30-day follow-up data are reported. RESULTS The patient group consisted of six girls and two boys (mean age 10.0, range 4.2-17.5 years): six children presented with moyamoya disease, two with moyamoya syndrome. We performed a one-staged revascularization of one MCA territory and both frontal areas in all patients. No significant complications occurred. Two patients experienced postoperative focal seizures, successfully treated with anti-epileptic medication. CONCLUSIONS The single-staged STA-MCA bypass with EDMS combined with bifrontal EDPS allowed revascularization of three regions (the MCA territory unilaterally and the frontal areas bilaterally) and may serve as an alternative and safe treatment option for pediatric moyamoya patients.
\end{abstract}

DOI: https://doi.org/10.1007/s00381-015-2665-y

Posted at the Zurich Open Repository and Archive, University of Zurich

ZORA URL: https://doi.org/10.5167/uzh-119743

Journal Article

Accepted Version

Originally published at:

Esposito, Giuseppe; Kronenburg, Annick; Fierstra, Jorn; Braun, Kees P J; Klijn, Catharina J M; van der Zwan, Albert; Regli, Luca (2015). "STA-MCA bypass with encephalo-duro-myo-synangiosis combined with bifrontal encephalo-duro-periosteal-synangiosis" as a one-staged revascularization strategy for pediatric moyamoya vasculopathy. Child's Nervous System, 31(5):765-772.

DOI: https://doi.org/10.1007/s00381-015-2665-y 
"STA-MCA bypass with encephalo-duro-myo-synangiosis combined with bifrontal encephalo-duroperiosteal-synangiosis" as a one-staged revascularization strategy for pediatric moyamoya vasculopathy.

Giuseppe Esposito $^{1}$; Annick Kronenburg ${ }^{2}$; Jorn Fierstra ${ }^{1}$; Kees P.J. Braun ${ }^{2}$; Catharina J.M. Klijn ${ }^{2}$, Albert van der Zwan $^{2}$, Luca Regli ${ }^{1}$.

1. Department of Neurosurgery, University Hospital Zurich, Zurich, Switzerland.

2. Department of Neurology and Neurosurgery, Brain Center Rudolf Magnus, UMCU Utrecht, the Netherlands.

\section{Affiliations and addresses of the authors:}

Giuseppe Esposito, MD, $\mathrm{PhD}$

Department of Neurosurgery, University Hospital Zurich, Zurich, Switzerland.

Frauenklinikstrasse 10 - CH-8091 Zürich, Switzerland

Annick Kronenburg, MD

Department of Neurology and Neurosurgery, Brain Center Rudolf Magnus, UMCU Utrecht, the Netherlands. Heidelberglaan 100, 3584 CX Utrecht, the Netherlands

Jorn Fierstra, MD, PhD

Department of Neurosurgery, University Hospital Zurich, Zurich, Switzerland.

Frauenklinikstrasse 10 - CH-8091 Zürich, Switzerland

Kees P. Braun, MD, PhD

Pediatric Neurology, Brain Center Rudolf Magnus, UMCU Utrecht, the Netherlands.

Heidelberglaan 100, 3508 AB Utrecht, the Netherlands

Catharina J.M Klijn, MD, PhD

Department of Neurology and Neurosurgery, Brain Center Rudolf Magnus, UMCU Utrecht, the Netherlands. Heidelberglaan 100, 3508 AB Utrecht, the Netherlands

Albert van der Zwan, MD, PhD

Department of Neurology and Neurosurgery, Brain Center Rudolf Magnus, UMCU Utrecht, the Netherlands. Heidelberglaan 100, 3508 AB Utrecht, the Netherlands

Luca Regli, MD

Department of Neurosurgery, University Hospital Zurich, Zurich, Switzerland.

Frauenklinikstrasse 10 - CH-8091 Zürich, Switzerland

\section{Corresponding author:}

Giuseppe Esposito, MD, PhD 
Department of Neurosurgery, University Hospital Zurich, Zurich, Switzerland Frauenklinikstrasse 10 - CH-8091 Zürich, Switzerland

Tel: +41-44-2551649 - Fax: +41-44-2554387 E-mail: giuseppe.esposito@usz.ch 


\section{ABSTRACT}

Purpose. Moyamoya vasculopathy progressively compromises cerebral blood flow resulting in chronic hypoperfusion. The middle cerebral artery (MCA) territory and the bifrontal areas are the regions most frequently affected. Although most techniques aim to only revascularize the MCA territory, augmentation of blood flow of the bifrontal areas is of importance in the pediatric moyamoya population since these regions play an important role in cognition, intellectual development, and in lower extremity and sphincter function. We recently described a one-staged surgical procedure combining revascularization of three regions, the MCA territory unilaterally and the frontal areas bilaterally. The purpose of this article is to report our surgical experience in eight children and to emphasize the rational for bifrontal revascularization.

Methods. We report a case series consisting of eight children where the following surgical strategy was applied: 1) a direct superficial temporal artery-to-middle cerebral artery (STA-MCA) bypass with encephalo-duro-myosynangiosis (EDMS) for unilateral MCA revascularization; in combination with 2) a bifrontal encephalo-duroperiosteal-synangiosis (EDPS) for bifrontal revascularization. Patients' characteristics and 30-day follow-up data are reported.

Results. The patient group consisted of six girls and two boys (mean age: 10.0, range 4.2-17.5 years): six children presented with moyamoya disease, two with moyamoya syndrome. We performed a one-staged revascularization of one MCA territory and both frontal areas in all patients. No significant complications occurred. Two patients experienced post-operative focal seizures, successfully treated with anti-epileptic medication.

Conclusions. The single-staged STA-MCA bypass with EDMS combined with bifrontal-EDPS allowed revascularization of three regions (the MCA territory unilaterally and the frontal areas bilaterally) and may serve as an alternative and safe treatment option for pediatric moyamoya patients.

Key words: bypass; cerebral revascularization; children; frontal area; moyamoya; pericranial flap. 


\section{Abbreviations}

ACA: anterior cerebral artery

CBF: cerebral blood flow

CVR: cerebrovascular reserve

CT: computed tomography

DSA: digital subtraction angiography

EDAS: encephalo-duro-arterio-synangiosis

EDMS: encephalo-duro-myo-synangiosis

EDPS: encephalo-duro-periosteal-synangiosis

EDS: encephalo-duro-synangiosis

EMS: encephalo-myo-synangiosis

EPS: encephalo-periosteal-synangiosis

ICA: internal carotid artery

IF: interhemispheric fissure

IQ: intelligence quotient

MCA: middle cerebral artery

MMD: moyamoya disease

MMS: moyamoya syndrome

MMV: moyamoya vasculopathy

MRA: magnetic resonance angiography

MRI: magnetic resonance imaging

PET: positron emission tomographic

SSS: superior sagittal sinus

STA: superficial temporal artery

STA-ACA: superficial temporal artery to anterior cerebral artery

STA-MCA: superficial temporal artery to middle cerebral artery

TIA: transient ischemic attack.

\section{Running title.}

One-staged unilateral MCA and bilateral frontal revascularization. 


\section{INTRODUCTION}

In patients with moyamoya vasculopathy (MMV), surgical revascularization is performed to prevent recurrent stroke and to stabilize or improve neurological outcome and quality of life.[1] Applied techniques of revascularization vary and it remains unclear whether one technique should be preferred over the other, both in adults and in children.[1-3] The most commonly used direct revascularization procedure is the superficial temporal artery to middle cerebral artery (STA-MCA) bypass that instantly augments blood supply to the middle cerebral artery (MCA) territory. Indirect techniques rely on the overlay of vascularized tissue (like the temporal muscle, pericranium and dura) onto the cerebral cortex in order to promote neoangiogenesis over time.[1-3] Combined revascularization procedures provide the advantages of both techniques.[2-7]

In addition to symptoms that can be ascribed to the MCA territory, children may present with lower extremity motor weakness and neuropsychological dysfunctions probably due to involvement of the frontal lobes.[8-12] In pediatric MMV, the cerebral blood flow (CBF) in the bifrontal areas as well as in the anterior watershed territory may continue to worsen despite good collateral formation or successful revascularization of the MCA territory.[9-12] Therefore, it is important to consider timely revascularization of the frontal areas, to prevent neurocognitive decline in pediatric patients.[8-12]

We recently published the technique of a one-staged surgical procedure for children diagnosed with MMV, which combines revascularization of three regions, the MCA territory unilaterally and the frontal areas bilaterally. This technique consists of unilateral STA-MCA bypass with encephalo-duro-myo-synangiosis (EDMS) and bifrontal encephalo-duro-periosteal-synangiosis (EDPS).[3]

The purpose of this article is to report our surgical experience in a series of eight pediatric moyamoya patients and to emphasize the rational for bifrontal revascularization.

\section{PATIENTS AND METHODS}

\section{Patients}

We retrospectively reviewed all pediatric patients with MMV who were operated on by performing the onestaged procedure (unilateral STA-MCA bypass with EDMS and bifrontal EDPS) between December 2009 and March 2012.

Pre-operative work-up includes Magnetic Resonance Imaging (MRI), 6-vessel digital subtraction angiography (DSA), $\mathrm{H}_{2} \mathrm{O}$-positron emission tomography (PET) and neuropsychological evaluation. These investigations are repeated one year after surgery.

According to our protocol, "unilateral STA-MCA bypass with EDMS combined with bifrontal EDPS in one step surgical procedure" was indicated for children presenting with both the following conditions: 1) hemodynamic compromise (impaired CBF and/or cerebrovascular reserve - CVR) or ischemic symptoms in a MCA territory; 2) hemodynamic compromise (impaired CBF and/or CVR) or ischemic symptoms in both frontal regions.

We registered clinical characteristics (disease, sex, age, pre-operative symptoms), surgical data (side and type of surgery, operative time), post-operative course and events (occurrence of complications, days of admittance, 30days follow-up). 
Unexpected events within 30 days after surgery are described as peri-operative complications. Episodic symptoms within 30 days postoperatively which resembled preoperative events in terms of frequency, duration and aspect (e.g. unchanged TIA's) were not included as peri-operative complications.

We report in details the treatment and follow-up data of a child affected by moyamoya disease (MMD).

\section{Description of the surgical technique}

\section{Direct (STA-MCA) and indirect (EDMS) bypass for unilateral MCA territory revascularization}

The patient is placed in supine position with the head mildly extended in the 3-points Mayfield headrest and $30^{\circ}$ rotated to the opposite side. Care has to be paid not to injure the contralateral STA as this would compromise a future STA-MCA bypass on the opposite side. We do not shave the hair.

The incision starts over the parietal branch of the STA. The STA-MCA bypass is performed according to the classic technique described elsewhere.[13] In brief, the parietal branch of the STA branch is dissected and prepared under microscopic view: this donor vessel is kept intact up to the anastomotic procedure. The temporal muscle is cut along the skin incision and a craniotomy is performed on the Sylvian point over a large cortical MCA branch. The dura mater is opened in a star shaped fashion preserving the main branches of the middle meningeal artery. After meticulous hemostasis, the dural flaps are reflected subdurally under the bone window, to obtain encephalo-duro-synangiosis (EDS) (figure $2 \mathrm{a}-\mathrm{b}$ ). The cortex is inspected for the largest cortical M4 recipient artery, which is dissected by means of arachnoid opening. A segment with no or only few cortical sidebranches is chosen (1-2 of the tiny side-branches may need to be interrupted). Two silicon triangle-shaped background-sheets are inserted beneath the recipient artery, in order to facilitate the construction of the anastomosis. A temporary non-traumatic microvascular clip is placed across the exposed STA proximally. The distal STA is cut in a fish-mouth to increase the opening diameter of the donor vessel and prepared for the micro-anastomosis. A blue dye is applied onto the donor and recipient vessels to improve visualization during the anastomotic procedure. Non-traumatic temporary microvascular clips are applied on the recipient vessel. A linear arteriotomy on the cortical recipient is performed so that the micro-anastomosis is at least 2.5 times the size of the diameter of the recipient vessel. Two 10-0 monofilament sutures are applied at the toe and the heel of the anastomotic site to anchor the donor and recipient vessel. The micro-anastomosis is performed with interrupted 10-0 monofilament sutures to allow anastomosis growth with time. Before knotting the last suture, the anastomosis is flushed to clear air. Flow is re-established by removing first the distal and then the proximal temporary clips on the cortical MCA recipient artery, and finally the clip on the proximal STA (Figure 2c). Bypass patency is assessed with Indocyanine green videoangiography (performed by the use of a commercially available microscope, OPMI ${ }^{\circledR}$ Pentero, Carl Zeiss Co, Oberkochen, Germany). The flow in the bypass is quantitatively assessed using an intra-operative flow probe (Transonic Systems Inc., Ithaca NY). Flow values over $15 \mathrm{ml} / \mathrm{min}$ are considered sufficient. In pediatric moyamoya patients, flow values are expected to be between 15 and $50 \mathrm{ml} / \mathrm{min}$, depending on patients' age, donor and recipient characteristics and hemodynamic conditions. The micro-anastomosis is observed for 20 minutes to make sure there is no decrease in flow. Closure is performed by covering the exposed cortex with the temporal muscle and by suturing the muscle to the dural 
edges (to obtain encephalo-myo-synangiosis - EMS). The bone flap is then secured into place above the muscle. Attention is paid to avoid any compression of the bypass (Figure $2 \mathrm{f}$ ).

\section{Bifrontal EDPS}

After completing the direct (STA-MCA) and the indirect (EDMS) bypass for a unilateral MCA territory revascularization, the skin incision is extended frontally $4 \mathrm{~cm}$ over the midline, staying behind the hairline (Figures 1a, 2a). This extension can also be made in a zig-zag fashion[14]. The scalp flap is reflected anteriorly and a vascularized bifrontal pericranial flap, consisting of the periosteum and the overlying loose areolar layer, is dissected (Figure $1 \mathrm{a}-\mathrm{b}, 2 \mathrm{~d}$ ). The pericranial flap is kept pediculated towards the bitemporal and biorbital regions to maximize vascular supply. This pediculated pericranial flap will serve to perform the bifrontal encephaloperiosteal-synangiosis (EPS). Two separate frontal parasagittal craniotomies $(4 \times 5 \mathrm{~cm})$ are performed: one on the left and one the right side. The craniotomies are localized $2 \mathrm{~cm}$ away from the midline to avoid injuring the superior sagittal sinus (SSS) and the parasagittal veins. The dura is then opened in a star shaped fashion and, after meticulous hemostasis, the dural flaps are inverted and reflected under the edges of each frontal bone window to obtain EDS (figures 1b, 2d). Small cortical arachnoidal openings are made. The periosteal flap is then positioned over the cortical convexity and sutured laterally to the dura (EPS) (Figures 1c, 2e). Both frontal bone flaps are repositioned and fixed (Figures 1d, 2e-f). The scalp flap is re-approximated and the skin incision closed in 2 layers taking care not to compromise the STA-MCA bypass.

\section{RESULTS}

\section{Case series}

Patient and operation characteristics, occurrence of complications and 30-day follow-up data are summarized in Table 1.

Between December 2009 and March 2012 we operated on eight pediatric moyamoya patients (6 girls; 2 boys) with the combined one-staged technique (Table 1). Mean age was 10.0 years (range 4.2-17.5). Six children were affected by moyamoya disease (MMD). Of the two patients with moyamoya syndrome (MMS), one patient had neurofibromatosis type I (case number 5) and the other trisomy 6 and a 17q25 translocation (case number 6). All patients were operated on by the same senior surgeon (L.R.) at the University Medical Center, Utrecht, The Netherlands.

The mean duration of the procedure (from incision to closure of the skin) was 6 hours and 54 minutes (range $5: 10-9: 35)$.

According to our protocol, we performed blood transfusions depending on the hemoglobin and hematocrit levels. In two children we adminstered transfusions peri-operatively (cases 5 and 7). In three other children, one-time blood transfusion was administered within 5 days postoperatively (case 1-3). The blood loss peri-operatively did not exceed $500 \mathrm{cc}$ in any patient. 
Two patients experienced complications. One patient (case number 2) had a 30-minute episode of focal seizures with twitching of the left corner of the mouth 7 hours postoperatively that was treated with Oxcarbazepine. CTscan showed diffuse edema in the treated right hemisphere, which resolved on follow-up neuroimaging. The other patient (case number 8) had short lasting symptoms (each lasting less than 10 minutes) on the fifth operative day consisting of sensibility disorders in his right arm and dysarthria. These symptoms were accompanied by aggressive behaviour, which he did not experience before. These symptoms partly resembled his pre-surgical events (before anti-epileptic drugs were prescribed) and were attributed to either TIAs or focal seizures. Valproic acid was restarted and symptoms resolved within 2 days. MRI revealed no new findings.

At follow-up, no wounds problems occurred and a good cosmetic outcome was achieved in all eight children.

\section{Illustrative case}

An 8-year-old previously healthy (case 3, Table 1) boy presented with multiple transient ischemic attacks (TIAs) over the span of one year. The TIAs consisted of symmetric leg weakness, dysphasia, right-sided hemiparesis and episodes of migraine, mostly following agitation and hyperventilation. The neurological examination showed no deficits. DSA revealed bilateral narrowing of the internal carotid artery termination including the M1 and A1 segments with presence of bilateral compensatory collaterals confirming MMD (Figure 3 left panel). The $\mathrm{H}_{2} \mathrm{O}$-PET scan showed evidence of decreased baseline CBF and impaired CVR to acetazolamide in both MCA territories and in the frontal areas bilaterally (Figure 3 right panel).

Based on the symptomatic course of the disease, the characteristics of the TIAs and the impaired CVR in the left MCA territory and bifrontal areas, we treated the patient with the described technique (Figure 1-2).

The post-operative course was uneventful. An early MRI examination at day 9 postoperatively did not reveal new ischemic lesions and MRA showed good patency of the bypass. Three TIAs were reported at 2-month follow-up (which resembled preoperative events in terms of duration and aspect). Thereafter no further ischemic episodes were reported and the boy remained asymptomatic for the next 10 months. At 6-months follow-up MRI/MRA showed no new ischemic lesions, stable of MMD and patency of the bypass. Repeat $\mathrm{H}_{2} \mathrm{O}-\mathrm{PET}$ after one year showed improvement of CBF and CVR in the left MCA territory and in the frontal areas bilaterally (Figure 3 right panel).

\section{DISCUSSION}

We report a case series consisting of 8 pediatric patients treated with a single stage direct STA-MCA with EDMS for unilateral revascularization of MCA territory, in combination with bifrontal EDPS for bilateral frontal revascularization [3]. The indication to perform the one-step combined revascularization procedure was to have hemodynamic compromise and clinical symptoms involving concurrently both a MCA territory and the bifrontal areas. No significant complications occurred.

Adequate CBF supply in the bifrontal areas is of importance, especially in pediatric patients with MMV.[3, 8, 9, $11,15-18]$ Cerebral ischemia in this region can in fact lead to lower extremity motor weakness and to intellectual and neuropsychological dysfunction.[11, 17-21] Although little is known about the natural history of MMV, 
steep decline of neurocognitive performance has been described in $44 \%$ of the pediatric population.[20] Furthermore, there is growing evidence that decreased CBF, especially in the frontal lobes, is correlated with diminished intelligence.[16] Most surgical approaches for MMV focus on increasing the blood supply to the MCA territory.[2, 3, 13, 22] In theory, by surgically restoring blood flow in the bifrontal areas, there may be a beneficial effect on neurocognitive performance.[12] A recent study analysed neurocognitive profiles pre- and postoperatively on 65 pediatric patients with MMV operated by means of a combination of indirect bypass procedures (unilateral encephalo-duro-arterio-synangiosis - EDAS - in 12 patients; bilateral EDAS in 11 patients, bilateral EDAS and bifrontal encephalo-galeal-periosteal synangiosis in 42 patients). This study demonstrated a retained intelligence quotient (IQ) and a significant improvement in performance IQ after surgery.[20] The benefits of bifrontal revascularization on long-term cognitive outcome in children with MMV, however, remain to be established in larger clinical trials.

\section{Advantages and disadvantages of the proposed technique}

The main advantage of this technique consisted of the possibility to revascularize in one-session 3 different vascular regions: the MCA territory unilaterally and the frontal areas bilaterally. The technique combined direct and indirect revascularization procedures: direct STA-MCA bypass increased flow immediately and EDMS promoted progressive neoangiogenesis over time in the MCA territory. Bifrontal EDPS aimed at inducing progressive neoangiogenesis over the frontal lobes bilaterally.

This technique also offered the advantage of revascularizing both frontal areas using two separate parasagittal frontal craniotomies, located $2 \mathrm{~cm}$ away from the midline SSS. This reduced the risk of injuries to the SSS and the parasagittal veins. Similarly, this technique avoided exposure and opening of the interhemispheric fissure (IF) and of the arachnoid membrane of the medial frontal lobes. Furthermore, the technique allowed inverting and reflecting dural flaps under the craniotomy edges expanding thereby the cortical coverage area for neoangiogenesis.

The scalp is not incised separately for this combined procedure. The skin incision is located behind the hairline and requires no shaving. The use of a single skin incision (either curvilinear or in a zig-zag fashion) and three craniotomies gave an excellent cosmetic result. Large craniotomy flaps or multiple burr holes might lead to less favourable cosmetic outcomes.

Finally, the proposed technique does not compromise eventual future contralateral MCA territory revascularization, as well as revascularization procedures in the posterior circulation territory.

EDPS may represent a very useful alternative to the existing indirect procedures for frontal lobe revascularization or to direct STA-ACA bypass for revascularization of frontal areas.[11] STA-ACA bypass is known to be technically challenging, especially in pediatric patiens. In fact the site of micro-anastomosis into the ACA territory needs a very distal preparation of the STA frontal branch and the cortical recipient of the ACA is generally also very small and often located in a sulcus. Furthermore, performing a direct STA-ACA bypass may be difficult in combination with a direct STA-MCA bypass.

The EDPS is technically easier and therefore should be feasible in most children with MMV. The use of frontal pericranial flaps to induce neoangiogenesis in patients with MMV has shown to be an effective technique.[6, 11, 15, 23-26] The choice of using the periosteum (frontal pericranium) for the bifrontal revascularization relies on 
the abundant blood supply, providing nourishment to the bone, and potentially promoting neoangiogenesis.[11] The frontal pericranium receives blood supply mainly from the supraorbital and supratrochlear arteries (as well as from frontal branches of the STA), emphasizing the importance of preparing the pericranial flap pediculated anteriorly over the orbits to preserve its vascular supply.[25, 27]

Although the one-stage combined revascularization technique represented a long surgical procedure, the immediate post-operative and short-term (within 30 days) follow-up of the reported patient-series indicated the feasibility and safety of this technique. Data on long term clinical, neuropsychological, radiological and hemodynamic follow-up of the whole case series is currently being collected.

The presented method for surgical revascularization in pediatric patients with MMV represents a modification of existing techniques $[11,15,23]$. Bifrontal EDPS by itself could also be used as a supplementary procedure in patients who already underwent previous revascularization procedures, in case of bifrontal hypoperfusion or progression of the MMV with symptoms referable to frontal lobe hypoperfusion. Bifrontal EDPS itself is doable by means of an incomplete bicoronal incision, from one superior temporal line to the contralateral one.

\section{CONCLUSIONS}

The novelty of this STA-MCA bypass with encephalo-duro-myo-synangiosis combined with bifrontal encephalo-duro-periosteal-synangiosis is the one-stage approach, combining direct and indirect revascularization techniques in three different vascular regions: the MCA territory unilaterally and the frontal areas bilaterally. Direct STA-MCA bypass and EDMS in the MCA territory achieves both an immediate flow augmentation and promotes neoangiogenesis over time; bifrontal EDPS aims at inducing neoangiogenesis on the frontal lobes bilaterally. Surgical risk is minimized by avoiding exposure of the superior sagittal sinus and opening of the interhemispheric fissure. In our series, no significant complications due to surgery expansion were encountered. Furthermore, bifrontal EDPS itself could be a valuable procedure in patients who already underwent other cerebral revascularization procedures and who present symptoms referable to frontal lobe hypoperfusion.

\section{Acknowledgments}

The authors thank Mr. Peter Roth (Department of Neurosurgery, University Hospital Zurich, Zurich, Switzerland) for the drawings in Figure 2. 

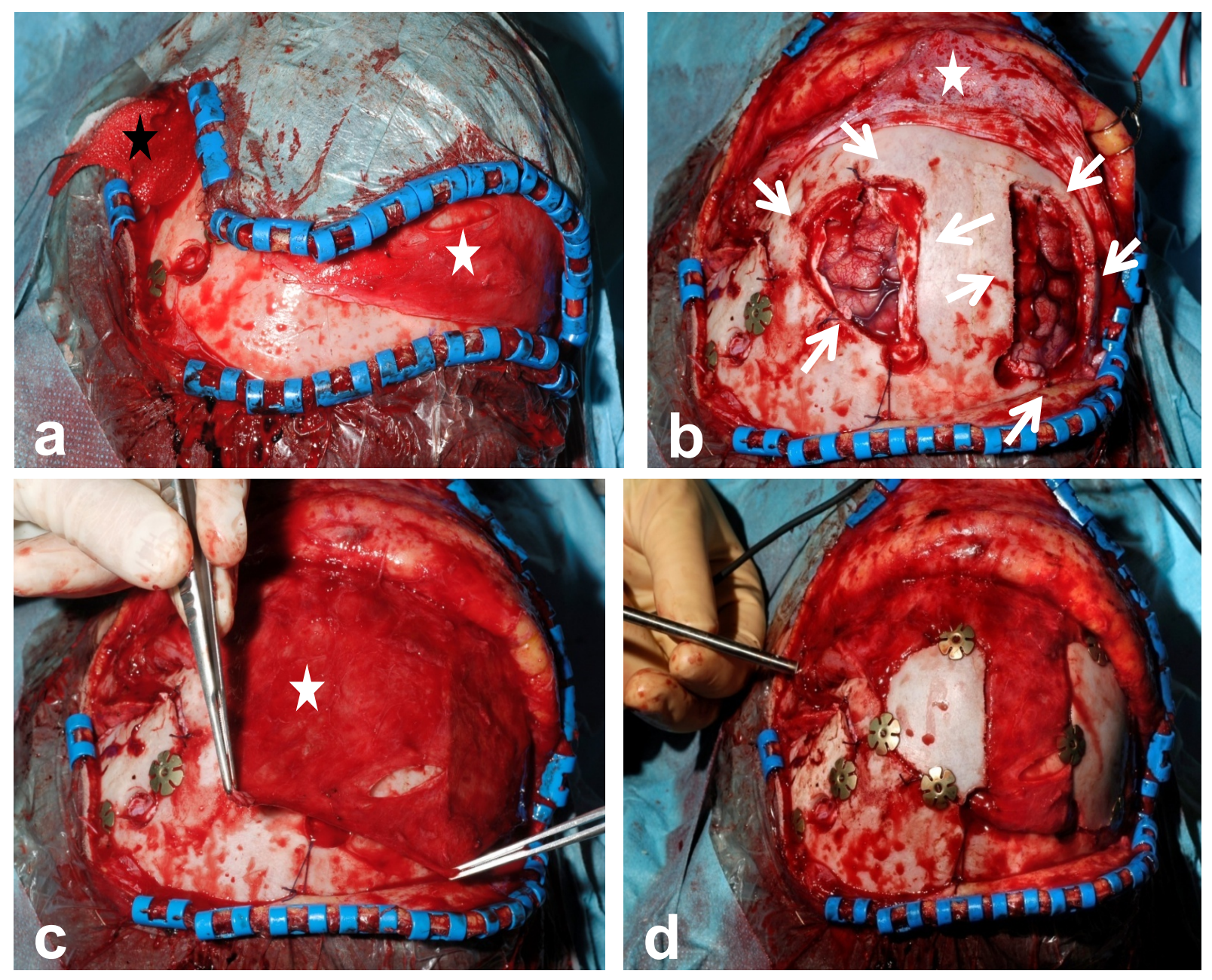

Figure 1

Intraoperative pictures:

a. After having performed a STA-MCA bypass and EDMS on the left side (the white arrow indicates the craniotomy and the black asterisk a cottonoid covering the bypass), the skin incision is extended 4-cm over the midline behind the hairline. The dissected frontal pericranial flap is indicated by the white asterisk (no-shaving technique).

b. The scalp flap is reflected anteriorly; the vascularized frontal pericranial tissue is dissected and reflected on the scalp flap (see white asterisk); two symmetric bilateral frontal parasagittal craniotomies are performed; the underlying frontal dura is then opened in a star-fashion and the dural flaps are inverted onto the cortex around each frontal bone window, to obtain EDS (see multiple white arrows).

c. The pericranial flap (white asterisk) is placed over the cortex surface and is sutured to dural edges to obtain EPS.

d. The bone flaps are repositioned. 


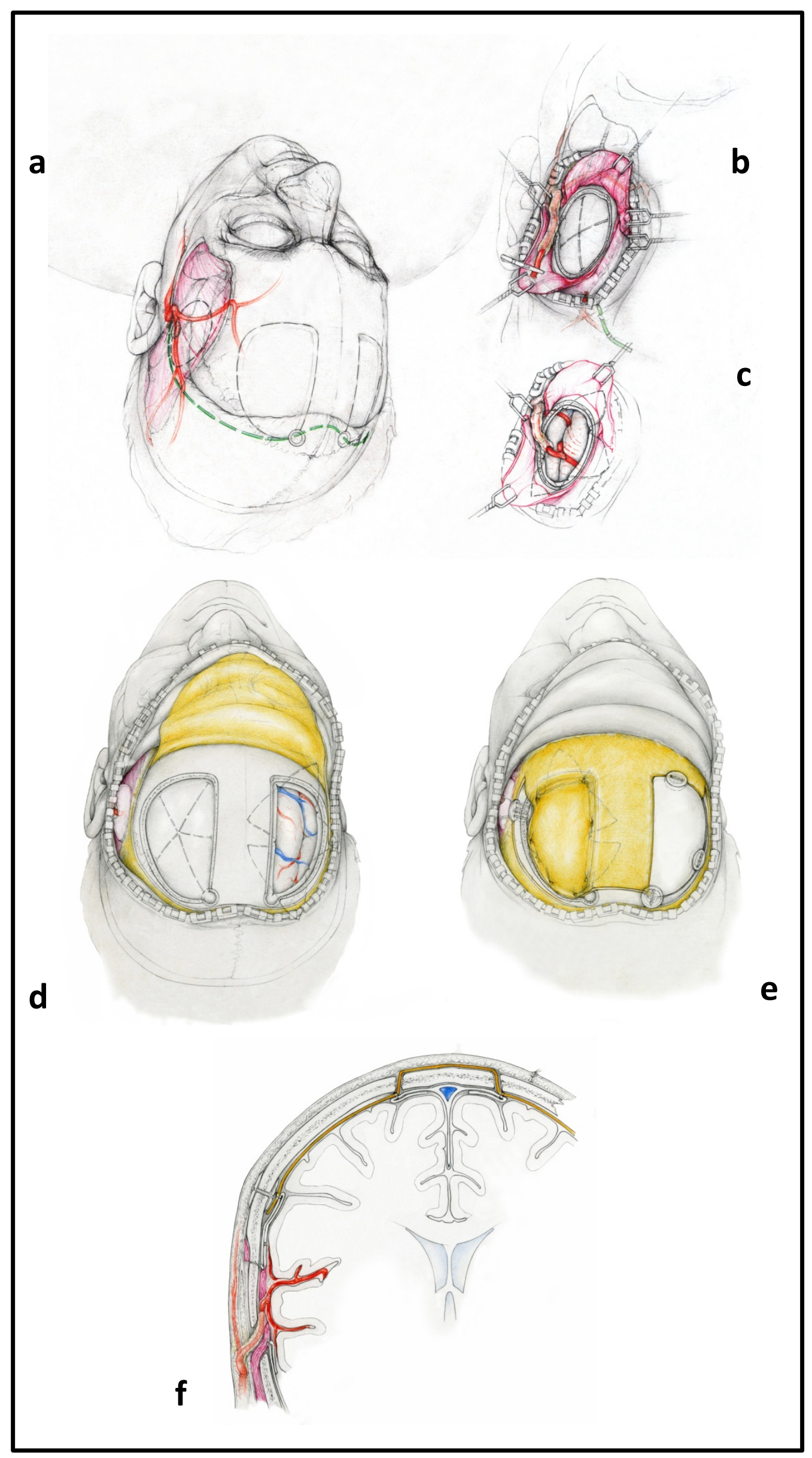




\section{Figure 2}

Sketch of the proposed technique:

a. The black temporal curvilinear dotted line represents the first skin incision performed to microsurgically isolate and prepare the donor vessel (i.e.: parietal branch of the STA). The craniotomy needed to perform the STA-MCA bypass with EDMS is also indicated by the circular black temporal dotted line. The skin incision is extended 4-cm over the midline, after the completion of the procedure for unilateral MCA revascularization (see green dotted line). All the skin incisions are performed behind the hairline. The area for the two subsequent frontal craniotomies is also illustrated.

b. After splitting the temporal muscle and performing a craniotomy over the Sylvian point, the dura is opened in a star-fashion way (see dotted lines). The donor vessel remains intact.

c. The dural flaps are inverted on the cortex around the bone window (see dotted lines) in order to obtain the EDS. Thereafter the STA-MCA bypass procedure is completed.

d. After having performed EMS (by covering the exposed cortex with the temporal muscle and by suturing the muscle to the dural edges), the bone flap is repositioned, the skin incision is then extended 4-cm over the midline behind the hairline. The scalp flap is then reflected anteriorly and the vascularized frontal pericranial tissue (in yellow) is dissected and reflected on the scalp flap. Two symmetric bilateral frontal parasagittal craniotomies are performed; the underlying frontal dura is opened in a starfashion and the dural flaps are inverted on the cortex around the frontal bone window, to obtain bifrontal EDS (see dotted lines).

e. To obtain bilateral frontal EPS, the pericranial flap is placed over the cortex surface and sutured to the dural edges bifrontally. The bone flaps are then repositioned.

f. This coronal section showing the 3 performed craniotomies ( 1 temporal, 2 frontal parasagittal); the STA-MCA bypass; the dura inverted on the cortex around each bone window (to obtain EDS); the temporal muscle laying on the exposed temporal cortex (to obtain EMS) and sutured to the dural edges; the pericranial flap placed over the frontal cortex surface (to obtain EPS) and anchored to the dural edges. 


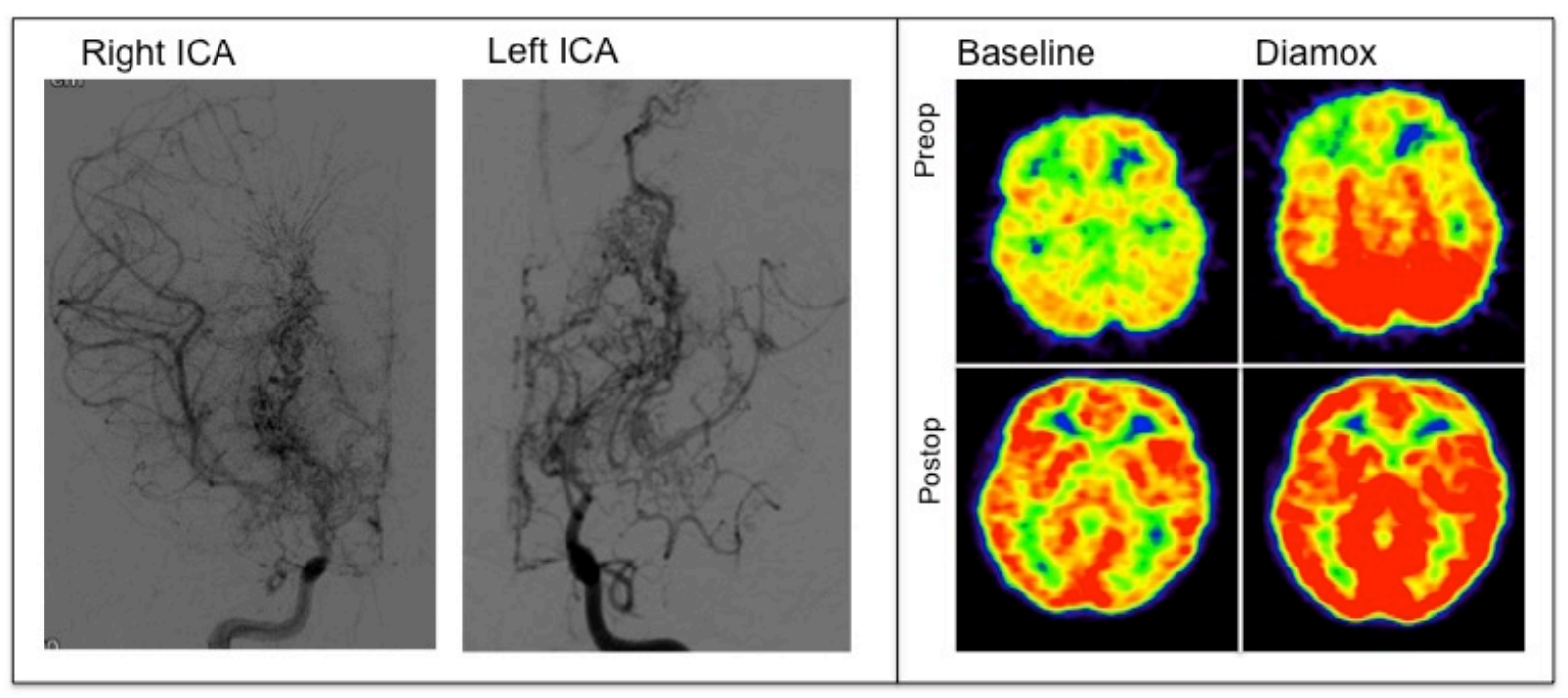

Figure 3

Left-panel: DSA images (antero-posterior view) showing narrowing of the ICAs termination including the M1 and A1 segment. Extensive hypertrophic lenticulo-striatal collaterals are evident bilaterally.

Right-panel: Pre-operative $\mathrm{H}_{2} \mathrm{O}$-PET before and after Diamox showing bilaterally the evidence of decreased $\mathrm{CBF}$ and haemodynamic failure in MCA territory and frontal areas bilaterally. One year post-operative $\mathrm{H}_{2} \mathrm{O}-\mathrm{PET}$ documenting a clear hemodynamic improvement in bifrontal areas and left MCA territory.

\section{REFERENCES}

[1] Scott RM, Smith ER (2009) Moyamoya disease and moyamoya syndrome. The New England journal of medicine 360: 1226-1237

[2] Kronenburg A, Braun KP, van der Zwan A, Klijn CJ (2014) Recent advances in moyamoya disease: pathophysiology and treatment. Current neurology and neuroscience reports 14: 423

[3] Kronenburg A, Esposito G, Fierstra J, Braun KP, Regli L (2014) Combined Bypass Technique for Contemporary Revascularization of Unilateral MCA and Bilateral Frontal Territories in Moyamoya Vasculopathy. Acta neurochirurgica Supplement 119: 65-70

[4] Kuroda S, Houkin K (2008) Moyamoya disease: current concepts and future perspectives. Lancet neurology 7: 1056-1066

[5] Baaj AA, Agazzi S, Sayed ZA, Toledo M, Spetzler RF, van Loveren H (2009) Surgical management of moyamoya disease: a review. Neurosurgical focus 26: E7

[6] Ishikawa T, Kamiyama H, Kuroda S, Yasuda H, Nakayama N, Takizawa K (2006) Simultaneous superficial temporal artery to middle cerebral or anterior cerebral artery bypass with pan-synangiosis for Moyamoya disease covering both anterior and middle cerebral artery territories. Neurologia medico-chirurgica 46: 462-468

[7] Houkin K, Ishikawa T, Yoshimoto T, Abe H (1997) Direct and indirect revascularization for moyamoya disease surgical techniques and peri-operative complications. Clinical neurology and neurosurgery 99 Suppl 2: S142-145 
[8] Festa JR, Schwarz LR, Pliskin N, Cullum CM, Lacritz L, Charbel FT, Mathews D, Starke RM, Connolly ES, Marshall RS, Lazar RM (2010) Neurocognitive dysfunction in adult moyamoya disease. Journal of neurology 257: 806-815

[9] Kim SK, Cho BK, Phi JH, Lee JY, Chae JH, Kim KJ, Hwang YS, Kim IO, Lee DS, Lee J, Wang KC (2010) Pediatric moyamoya disease: An analysis of 410 consecutive cases. Annals of neurology 68: 92-101

[10] Ibrahimi DM, Tamargo RJ, Ahn ES (2010) Moyamoya disease in children. Child's nervous system : ChNS : official journal of the International Society for Pediatric Neurosurgery 26: 1297-1308

[11] Park JH, Yang SY, Chung YN, Kim JE, Kim SK, Han DH, Cho BK (2007) Modified encephaloduroarteriosynangiosis with bifrontal encephalogaleoperiosteal synangiosis for the treatment of pediatric moyamoya disease. Technical note. Journal of neurosurgery 106: 237242

[12] Weinberg DG, Rahme RJ, Aoun SG, Batjer HH, Bendok BR (2011) Moyamoya disease: functional and neurocognitive outcomes in the pediatric and adult populations. Neurosurgical focus 30: E21

[13] Khan NR, Regli L. (2011) STA-MCA microanastomosis: surgical technique. In: Abdulrauf S (ed) Cerebral revascularization: techniques in extracranial-to-intracranial bypass surgery. Saunders/ Elsevier, Philadelphia, pp 93-97

[14] Leach P, Rutherford S, Likhith A, Leggate J (2004) Zig-zag bicoronal scalp incision for cranio-facial cases in paediatric neurosurgery. Child's nervous system : ChNS : official journal of the International Society for Pediatric Neurosurgery 20: 483-484

[15] Kim SK, Wang KC, Kim IO, Lee DS, Cho BK (2002) Combined encephaloduroarteriosynangiosis and bifrontal encephalogaleo(periosteal)synangiosis in pediatric moyamoya disease. Neurosurgery 50: 88-96

[16] Kuroda S, Houkin K, Ishikawa T, Nakayama N, Ikeda J, Ishii N, Kamiyama H, Iwasaki Y (2004) Determinants of intellectual outcome after surgical revascularization in pediatric moyamoya disease: a multivariate analysis. Child's nervous system : ChNS : official journal of the International Society for Pediatric Neurosurgery 20: 302-308

[17] Ishikawa $\mathrm{T}$, Houkin K, Kamiyama H, Abe H (1997) Effects of surgical revascularization on outcome of patients with pediatric moyamoya disease. Stroke; a journal of cerebral circulation 28: 1170-1173

[18] Ogawa A, Yoshimoto T, Suzuki J, Sakurai Y (1990) Cerebral blood flow in moyamoya disease. Part 1: Correlation with age and regional distribution. Acta neurochirurgica 105: 30-34

[19] Karasawa J, Touho H, Ohnishi H, Miyamoto S, Kikuchi H (1992) Long-term followup study after extracranial-intracranial bypass surgery for anterior circulation ischemia in childhood moyamoya disease. Journal of neurosurgery 77: 84-89

[20] Lee JY, Phi JH, Wang KC, Cho BK, Shin MS, Kim SK (2011) Neurocognitive profiles of children with moyamoya disease before and after surgical intervention. Cerebrovascular diseases 31: 230-237

[21] Ishii R, Takeuchi S, Ibayashi K, Tanaka R (1984) Intelligence in children with moyamoya disease: evaluation after surgical treatments with special reference to changes in cerebral blood flow. Stroke; a journal of cerebral circulation 15: 873-877

[22] Khan N, Schuknecht B, Boltshauser E, Capone A, Buck A, Imhof HG, Yonekawa Y (2003) Moyamoya disease and Moyamoya syndrome: experience in Europe; choice of revascularisation procedures. Acta neurochirurgica 145: 1061-1071; discussion 1071

[23] Kim CY, Wang KC, Kim SK, Chung YN, Kim HS, Cho BK (2003) Encephaloduroarteriosynangiosis with bifrontal encephalogaleo(periosteal)synangiosis in the 
pediatric moyamoya disease: the surgical technique and its outcomes. Child's nervous system : ChNS : official journal of the International Society for Pediatric Neurosurgery 19: 316-324 [24] Kim DS, Kye DK, Cho KS, Song JU, Kang JK (1997) Combined direct and indirect reconstructive vascular surgery on the fronto-parieto-occipital region in moyamoya disease. Clinical neurology and neurosurgery 99 Suppl 2: S137-141

[25] Kuroda S, Houkin K, Ishikawa T, Nakayama N, Iwasaki Y (2010) Novel bypass surgery for moyamoya disease using pericranial flap: its impacts on cerebral hemodynamics and long-term outcome. Neurosurgery 66: 1093-1101; discussion 1101

[26] Kinugasa K, Mandai S, Tokunaga K, Kamata I, Sugiu K, Handa A, Ohmoto T (1994) Ribbon enchephalo-duro-arterio-myo-synangiosis for moyamoya disease. Surgical neurology 41: 455-461

[27] Yoshioka N, Rhoton AL, Jr. (2005) Vascular anatomy of the anteriorly based pericranial flap. Neurosurgery 57: 11-16; discussion 11-16 


\begin{tabular}{|c|c|c|c|c|c|c|c|c|}
\hline $\begin{array}{l}\text { Case } \\
\mathrm{n}^{\circ}\end{array}$ & Sex & MMD/MMS & $\begin{array}{l}\text { Age (yrs) } \\
\text { at surgery }\end{array}$ & Preoperative events & Side surgery & Peri-operative complications $<30$ days* & $\begin{array}{l}\text { Operating time } \\
\text { (hrs;min) }\end{array}$ & $\begin{array}{l}\text { Days of } \\
\text { admittance }\end{array}$ \\
\hline 1 & $\mathrm{~F}$ & MMD & 4.2 & $\begin{array}{l}\text { Infarction L hemisphere; } \\
\text { TIAs R hemisphere }(<10 \mathrm{~min})\end{array}$ & Left (+ BF) & None & $05: 30$ & 14 days \\
\hline 2 & $\mathrm{~F}$ & MMD & 4.9 & TIAs R hemisphere $(<1 \mathrm{hr})$ & Right (+ BF) & $\begin{array}{l}7 \text { hrs postoperatively: Partial seizures } \mathrm{R} \\
\text { hemisphere (CT:edema,no infarction; } \\
\text { oxcarbazepine prescribed) }\end{array}$ & 07:53 & 10 days \\
\hline 3 & $M$ & MMD & 8.4 & $\begin{array}{l}\text { TIAs L hemisphere and } \\
\text { symmetric leg weakness (min- } \\
\text { 1hr); migraine }\end{array}$ & Left $(+B F)$ & None & 05:10 & 11 days \\
\hline 4 & $\mathrm{~F}$ & MMD & 7.9 & TIAs L hemisphere (min-1hr) & Left $(+B F)$ & None & $06: 23$ & 8 days \\
\hline 5 & $\mathrm{~F}$ & MMS & 9.7 & $\begin{array}{l}\text { TIAs R hemisphere }(\min -1 \mathrm{hr}) \text {; } \\
\text { migraine }\end{array}$ & Right (+ BF) & None & 05:33 & 9 days \\
\hline 6 & $\mathrm{~F}$ & MMS & 9.7 & $\begin{array}{l}\text { Collapses }(10-30 \mathrm{~min}) \\
\text { bilateral TIAs and infarction } \\
\text { (recent L) }\end{array}$ & Left (+ BF) & None & $07: 40$ & 7 days \\
\hline 7 & $\mathrm{~F}$ & MMD & 9.6 & $\begin{array}{l}\text { Severe cognitive impairment; } \\
\text { dyskinesia R hand }\end{array}$ & Left (+ BF) & None & $09: 35$ & 9 days \\
\hline 8 & $M$ & MMD & 17.5 & $\begin{array}{l}\text { Stuttering infarction and parzial } \\
\text { seizures } L \text { hemisphere } \\
\text { (temporarily valproic acid } \\
\text { prescribed) }\end{array}$ & Left (+ BF) & $\begin{array}{l}6 \text { days postoperatively: } 2 \text { TIAs/Partial } \\
\text { seitures } L \text { hemisphere (<10 min;MRI no } \\
\text { new findings; valproic acid restarted) }\end{array}$ & $07: 28$ & 6 days \\
\hline
\end{tabular}

Table 1: Patient and operation characteristics, occurrence of complications and 30-day follow-up.

$\mathrm{BF}=$ Bifrontal; $\mathrm{F}=$ female; hrs=hours; $\mathrm{L}=\mathrm{left}$; $\mathrm{M}=$ male; $\mathrm{min}=$ minutes; $\mathrm{MMD}=$ Moyamoya disease; no=number; $\mathrm{R}=$ right; $\mathrm{yrs=years}$ 